\title{
Diverse roles of macrophage polarization in aortic aneurysm: destruction and repair
}

(.) CrossMark

\author{
Zhao Cheng ${ }^{1}$, Yang-zhao Zhou ${ }^{2}$, Yin Wư ${ }^{2}$, Qi-ying Wu ${ }^{2}$, Xiao-bo Liao ${ }^{2}$, Xian-ming Fu² and Xin-min Zhou ${ }^{2}$
}

\begin{abstract}
Aortic aneurysm (AA) is defined as an enlargement of the aorta greater than 1.5 times its normal size. Early diagnosis of AA is challenging and mortality of AA is high. Curative pharmacological treatments for AA are still lacking, highlighting the need for better understanding of the underlying mechanisms of AA progression. Accumulating studies have proven that the polarization state of circulating monocyte-derived macrophages plays a crucial role in regulating the development of AA. Distinct macrophage subtypes display different functions. Several studies targeting macrophage polarization during AA formation and progression showed potential treatment effects. In this review, we focus on the recent advances of research on macrophage polarization in the progression of AA and propose that targeting macrophage polarization could hold great promise for preventing and treating AA.
\end{abstract}

Keywords: Macrophage polarization, Aortic aneurysm, Inflammation

\section{Introduction}

Aortic aneurysm (AA) is a dilatation of the aorta and is associated with severe complications, such as aortic rupture. Although significant progresses have been made in both open and endovascular surgery, curative pharmacological treatments for this condition, especially for inoperative small AA (diameter less than $5.5 \mathrm{~cm}$ ) are still lacking [1-3]. The underlying mechanisms involved in the formation and progression of AA have not been fully elucidated but are increasingly recognized to involve chronic inflammatory response, extracellular matrix (ECM) degradation, and matrix metalloproteinase (MMP) upregulation. Various types of inflammatory cells, including dendritic cells, B cells, $\mathrm{T}$ cells, neutrophils, mast cells, and macrophages, have been identified in both human and experimental AA tissues and have been shown to be associated with increased levels of proinflammatory cytokines and diverse proteases that contribute to AA development [4-9]. Of them, macrophages have been demonstrated to play a critical role in the formation and progression of AA. The two major

\footnotetext{
*Correspondence: xianmingfu@csu.edu.cn

${ }^{2}$ Department of Cardiovascular Surgery, The Second Xiang-ya Hospital, Central South University, Changsha, Hunan, People's Republic of China Full list of author information is available at the end of the article
}

macrophage phenotypes are known as classically-activated M1 macrophages (proinflammatory phenotype) and alternative activated M2 macrophages (anti-inflammatory phenotype) [10-12]. The main characteristic of M1 macrophages is the production of proteolytic enzymes and pro-inflammatory cytokines, such as tumor necrosis factor (TNF), Interleukin 6 (IL-6), IL-12, IL-1 $\beta$, nitric oxide synthase 2 (iNOS), Chemokine (C-X-C motif) ligand 9 (CXCL9) and the CXCL10. In contrast, M2 macrophages participate in the anti-inflammatory response through secretion of factors such as IL-10 or transforming growth factor- $\beta$ (TGF- $\beta$ ) and are involved in ECM remodeling and tissue repair [10, 13]. Recent findings have shown distinct macrophage subsets present in human and experimental murine AA tissues and are known to have different functions in the progression of AA. In this review, we provide current knowledge of the roles of distinct macrophage subsets on the development of AA, and particularly highlight their potential translational applications.

\section{The current treatments for aortic aneurysm}

$\mathrm{AA}$ is an asymptomatic but life-threatening aortic disease and constitutes the 13th most common cause of death in the U.S. In developing countries such as China, as the population ages and dietary pattern changes the 
morbidity of AA is gradually increasing. Along with the diagnostic imaging technology progresses, the diagnostic rate of AA is also increasing [14]. In the current guidelines for AA treatment, for AA of diameter $\geq 5.5 \mathrm{~cm}$, surgical resection, artificial vascular replacement or endoluminal stent repair are recommended. On the other hand, for small AA of diameter $<5.5 \mathrm{~cm}$, the aortic dilation rate is slower, and the risk of aortic rupture is reduced; thus, traditional surgical treatment or endoluminal stent repair do not show obvious benefits. For patients with small AA, periodical imaging examinations to monitor the AA diameter are recommended; once the AA diameter reaches $5.5 \mathrm{~cm}$ or the dilatation rate of AA is higher than $0.5 \mathrm{~cm}$ per year, optional surgical repair or endoluminal stent repair would be considered. In summary, all current treatments for AA dependent on mechanical intervention. However, when most AAs are detected, they are below the threshold for repair, leading to a significant observation period during which there is currently no non-surgical therapy to prevent or slow AA expansion [15], highlighting a real need for better understanding of the potential mechanisms involved in the pathogenesis of AA.

\section{Macrophage polarization}

Macrophages can be differentiated from circulating monocytes, bone marrow $\mathrm{CD} 34^{+} / \mathrm{CD} 86^{+}$hematopoietic stem cells and $\mathrm{CD} 25^{+} / \mathrm{CD} 44^{+} / \mathrm{FcR}^{+}$early $\mathrm{T}$ lymphocytes under appropriate conditions and can be distributed to various types of tissues $[16,17]$. Most macrophages originate from circulating monocytes, which extravasate out of blood vessel and accumulate in the injured or inflammatory sites [18]. Macrophages are essential for the innate immune system. The main functions of macrophages are phagocytosis, antigen presentation, and cytokine secretion, which play important roles in inflammation, immune defense, tissue reconstruction, metabolism, and the maintenance of homeostasis [19, 20]. Under certain microenvironments or stimulation by certain factors, macrophages can be categorized as various phenotypes, such as "classically activated" M1 macrophages and "alternatively activated" M2 macrophages. M1 macrophages are effector cells of Th1 cells and can be induced from innate macrophages stimulated by interferons (IFNs), microbial lipopolysaccharide (LPS), or other cytokines, such as tumor necrosis factor (TNF), and macrophage colony-stimulating factor (M-CSF). These factors induce local pro-inflammatory microenvironments, which lead to the expression of CD68+, $\mathrm{CD} 86+$, and $\mathrm{MHCII}+$ on macrophages and induce the overexpression of nicotinamide adenine dinucleotide phosphate(NADPH)-peroxidase and iNOS as well as the production of pro-inflammatory cytokines to perform efficient antigen presentation and eliminate antigens. Once M1 macrophages are consistently induced and activated, they may cause tissue damage. The phenotype of M2 macrophages is characterized as CD68+ and CD163+, and they can be induced by Th2 cytokines [10, 21, 22]. M2 macrophages can be sub-classified as M2a (stimulated by IL4 or IL13), M2b (stimulated by immune complexes in combination with IL-1 $\beta$ or LPS), M2c (stimulated by IL-10, transforming growth factor- $\beta$ [TGF- $\beta$ ] or glucocorticoids) and M2d (stimulated by TLR and adenosine A2A receptor agonists). Recently, new phenotypes of macrophages were described to result from additional stimuli under certain microenvironments. These include hemorrhage-associated macrophages called Mhem (induced by hemoglobin) [23], macrophages stimulated with oxidized phospholipids (Mox) [24], and M4 macrophages induced by chemokine ligand 4 [25], tumor-associated macrophages (TAMs) etc. The currently available data indicate that macrophage polarization is a multifactorial process in which a large number of factors can be involved, producing different activation scenarios (Fig. 1). Once a macrophage adopts a phenotype, it retains the ability to continue changing in response to new environmental influences. The reversibility of polarization, also called functional adaptability, has critical therapeutic value, especially in diseases where the M1/M2 imbalance has a pathogenic role such as autoimmune diseases or chronic inflammation-related diseases. Key transcription factors are clearly associated with macrophage polarization, such as the signal transducer and activator of transcription family (STATs), peroxisome proliferator-activated receptor (PPAR), cAMP response element binding protein (CREB)-CCAAT/ enhancer binding protein (C/EBP), hypoxia-inducible factors (HIF), nuclear factor kappa $B(N F \kappa B)$ and interferon regulatory factors (IRF) [26, 27].

\section{Macrophage polarization in the development of aortic aneurysm}

Previous studies have shown that the main pathological features of AA include inflammatory cells infiltration, apoptosis of vascular smooth muscle cells (VSMCs), Upregulation of MMPs, and ECM degeneration. Inflammatory cells such as macrophages can infiltrate into aortic tissue and secrete MMPs and pro-inflammatory factors to promote destruction of ECM and induce apoptosis of VSMCs, resulting in AA formation. Studies have demonstrated that aortic injuries could induce the migration of bone marrow-derived cells to injury sites, where they differentiate into macrophages or fibroblasts, to participate in the immune response, repair and reconstruction of aortic wall $[16,17]$. This phenomenon suggests 


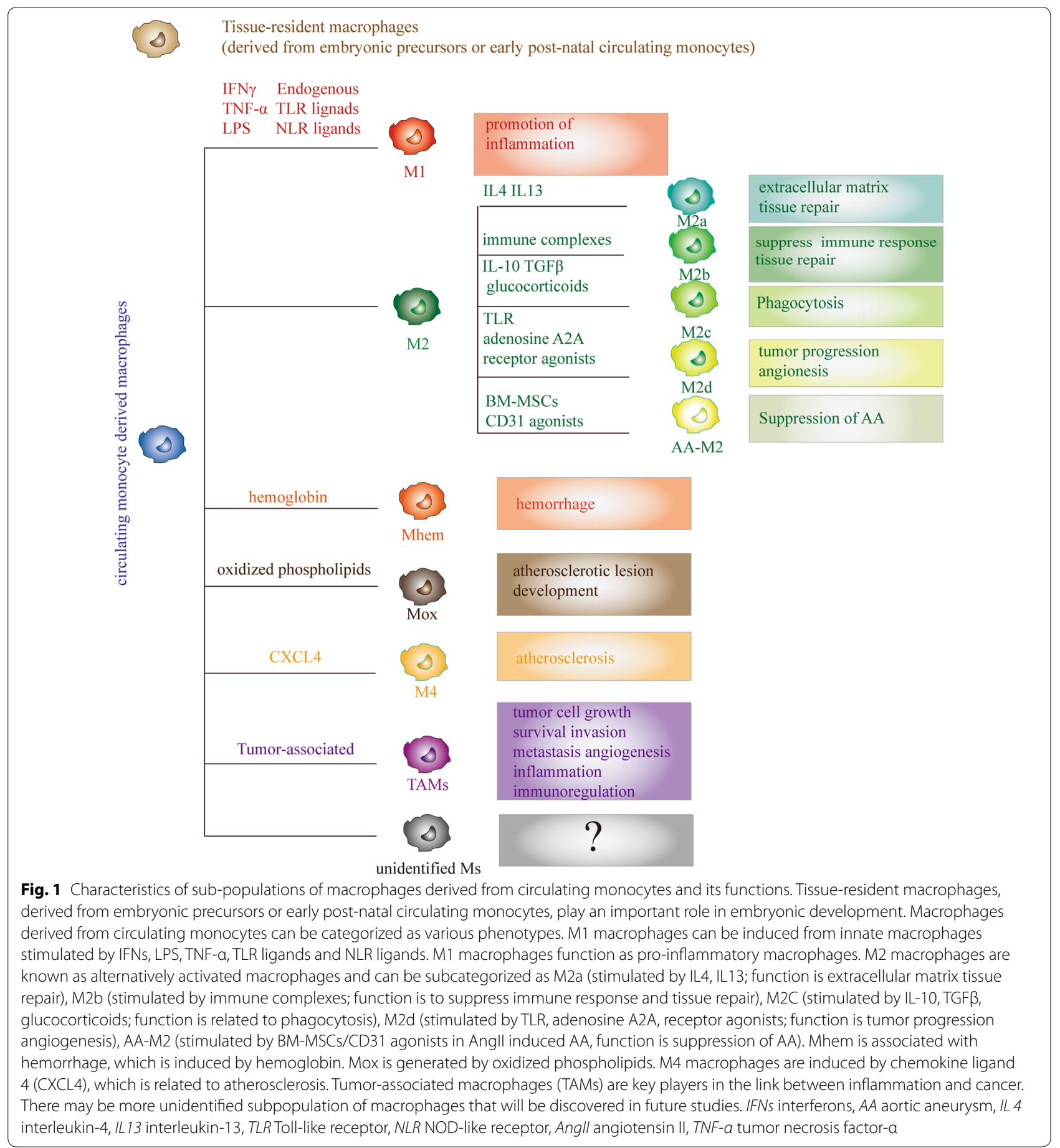

that macrophages originating from bone marrow cells can function to repair the injured aortic tissues.

Numerous recent studies have suggested that M1 and M2 polarization of macrophages may be involved in the aortic remodeling and development of AA [18]. Moore et al. [19] reported that hypertension in mice with angiotensin (Ang II) infusion is associated with accumulation of Ly6C $\mathrm{C}^{\text {hi }}$ monocytes in the aortic tissues. These cells differentiate into M2 macrophages, which likely promote ECM remodeling, including collagen deposition and elastin loss. Using laser capture microdissection (LCM) and immunohistochemistry, Boytard et al. [20] observed the distribution of subtypes of macrophages in human AA tissues. They found that M1 macrophages 
$(\mathrm{CD} 68+\mathrm{MR}-)$ were predominant in the adventitia, while M2 macrophages (CD68+MR+) were predominant in the intraluminal thrombus. They also found that stabilin 1 , involved in the uptake and degradation of unwanted molecules, was overexpressed in M2 (CD68+MR+) macrophages, which suggested that M1 and M2 macrophages may play different roles in AA development. Hans et al. [21] reported that Notch1 haploinsufficiency prevents the influx of proinflammatory macrophages (M1) at the aneurysmal site by causing defects in macrophage migration and proliferation. Decreased levels of Notch1 protects against the formation of AA by preventing macrophage recruitment and attenuating the inflammatory response in the aorta. Hasan et al. [22] found that M1 and M2 macrophages are present in equal proportions in unruptured aneurysms. However, there is an increase in M1 macrophages and mast cells in ruptured aneurysms. A macrophage M1/M2 imbalance and upregulation of mast cells may play roles in the progression of cerebral aneurysms to rupture. Batra et al. [23] reported that IL (interleukin)-1 $\beta$ was differentially expressed in human plasma from patients with AA compared with matched atherosclerotic controls. Wang et al. [24] found that TNF-stimulated gene-6 (TSG-6) was elevated in both the plasma and aortic wall of patients with AA compared with healthy and risk-factor matched non-AA donors. Both IL- $1 \beta$ and TSG- 6 are related with the regulation of macrophage polarization in AA. In our primary study, we observed that, at early stages of AngII induced AA in Apo $\mathrm{E}^{-/-}$mice, M1 macrophages were increased along with secretion of several pro-inflammatory cytokines and chemokines. After AA formation, M2 macrophages markedly increased along with secretion of anti-inflammatory cytokines and chemokines.

Based on the above evidence, distinct macrophage subtypes are believed to regulate aortic remodeling after injury (Table 1). At the early stage of AA, M1 macrophages dominate at the site of injured aortic tissues function in inflammatory factors expression, proteolysis, and phagocytosis. At late stage, M2 macrophages accumulate preferentially and facilitate reparative processes such as ECM deposition and angiogenesis. Maintenance of an appropriate M1/M2 ratio is crucial for aortic tissue homeostasis (Fig. 2). Understanding the role of macrophage polarization in aortic remodeling may lead to the development of novel therapies for AA.

\section{Modulating macrophage function for treatment of aortic aneurysm}

Macrophage polarization can regulate inflammation, thus affecting the development and prognosis of inflammation-related diseases. Recent studies have shown that using drugs or cell therapy modulating macrophage polarization could achieve therapeutic effects for AA [25-27]. Moran et al. [25] showed that systemic administration of the rapamycin inhibitor everolimus limits $\mathrm{AA}$ in the AngII-infused $\mathrm{ApoE}^{-1-}$ mouse model via suppressed development of bone marrow CCR2 monocytes, reduced egress of these cells into the circulation and diminished IFNy/lipopolysaccharide-stimulated M1 polarization in bone marrow monocyte-differentiated macrophages. In Yoshihara et al. [26] study, the AA model was developed by AngII infusion in $\mathrm{ApoE}^{-/-}$mice. AA formation and macrophage infiltration were significantly suppressed after Eicosapntemacnioc Acid (EPA) and Docosahexaenoic acid (DHA) administration. The expression of arginase 2, a marker of pro-inflammatory macrophages (M1), was significantly lower, and that of Ym1, a marker of anti-inflammatory macrophages (M2), was significantly higher after EPA and DHA administration. Pope et al. [27] reported that administration of D-series resolvins could attenuate murine AA formation by increasing M2 macrophage polarization and altering inflammatory cytokine expression. Dale et al. [28] showed that treatment of bone marrow-derived macrophages with Elastin-derived peptides (EDPs) could induce M1 macrophage polarization. Injection of M2-polarized macrophages reduced aortic dilation after

Table 1 Macrophage subtypes involved in aortic remodeling and aneurysm

\begin{tabular}{|c|c|c|}
\hline Disease modle/objects & Pathway/key regulators & References \\
\hline Ang II-infused ApoE ${ }^{-/-}$mice & M2 macrophage accumulation in the aortic wall & Moore et al. [19] \\
\hline Human aneurysmal infrarenal aortic wall & $\begin{array}{l}\text { M1 macrophage was predominant in the adventitia while the } \mathrm{M} 2 \text { macrophages in the } \\
\text { intraluminal thrombus/stabilin } 1\end{array}$ & Boytard et al. [20] \\
\hline Ang II-infused ApoE $E^{-/-}$mice & Notch1 & Hans et al. [21] \\
\hline Cerebral aneurysms & Macrophage M1/M2 imbalance and upregulation of mast cells & Hasan et al. [22] \\
\hline Patients with $\mathrm{AA}$ & IL-1 $\beta$ was differentially expressed in human plasma with in patients with $A A$ & Batra. et al. [23] \\
\hline Patients with AA & $\begin{array}{l}\text { NF-stimulated gene-6 (TSG-6) elevated in both the plasma and aortic wall of patients } \\
\text { with AA }\end{array}$ & Wang et al. [24] \\
\hline
\end{tabular}

Angll angiotensin II, IL-1 $\beta$ interleukin-1 $\beta, A p o E$ apolipoprotein $\mathrm{E}, A A$ aortic aneurysm, TSG- 6 tumor necrosis factor-inducible gene 6 


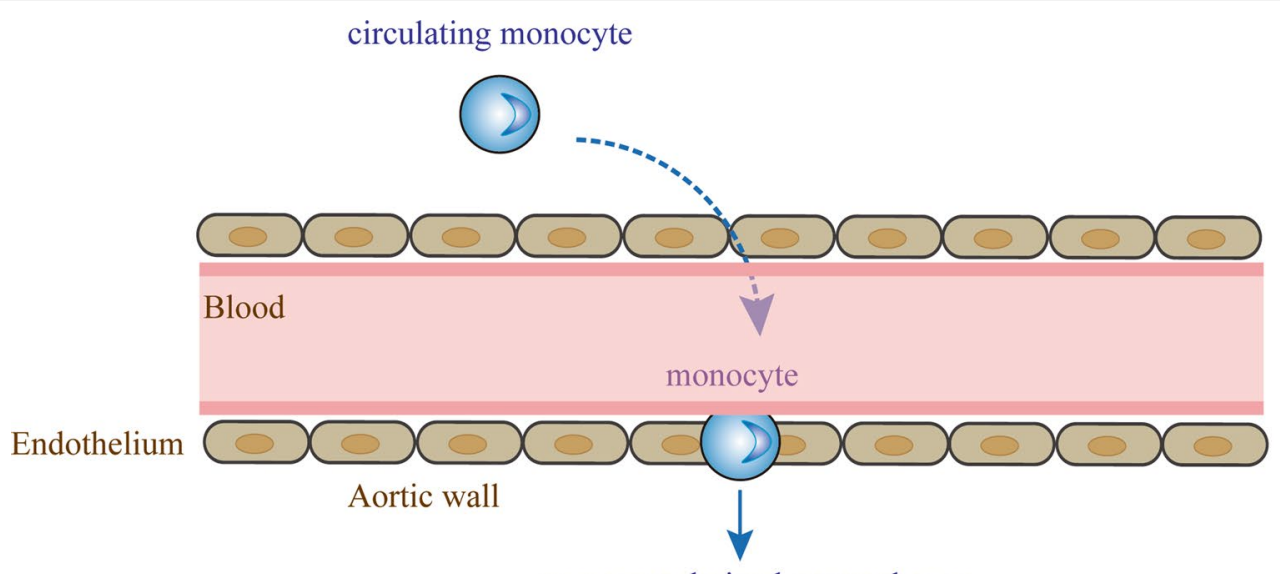

monocyte derived macrophages
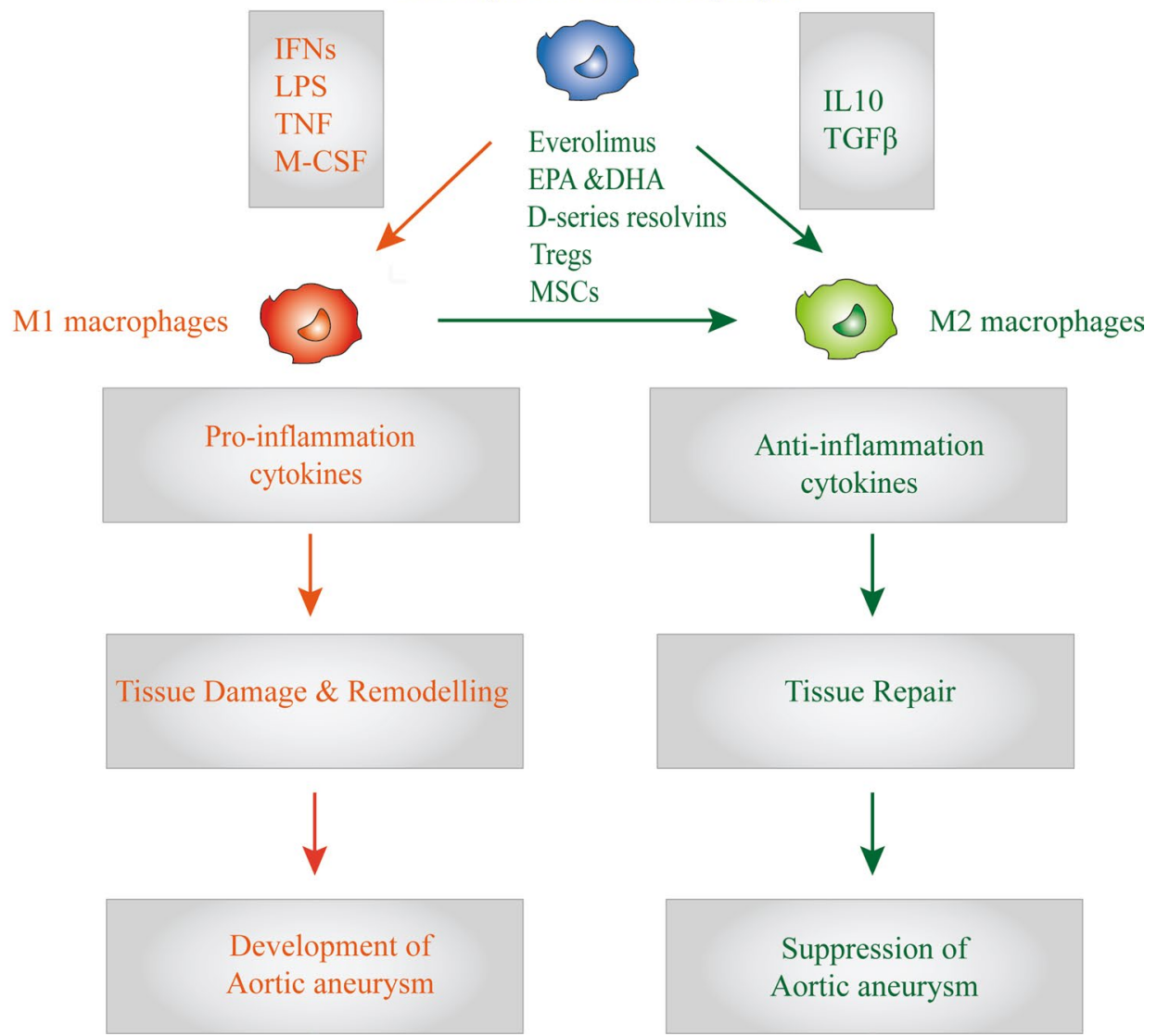

Fig. 2 Macrophage polarization in AA and its functions. M1 and M2 polarization of macrophages derived from circulating monocytes were found to be closely related with the development and prognosis of AA. The chronic inflammatory microenvironments at the site of AA, such as the expression of IFNs, LPS, TNF and M-CSF, induce M1 macrophages, which function as pro-inflammatory macrophages; in response to inflammation, they can cause tissue damage related to the development of AA. On the other hand, M2 macrophages induced by IL-10 and TGF $\beta$ can function as anti-inflammatory macrophages, which play a key role in tissue remodeling. Some molecules or factors have been reported to induce the transformation of M1 macrophages into M2 macrophages, such as Everolimus, EPA \& DHA, D-series resolvins, Tregs and MSCs. Angll angiotensin II, $A A$ aortic aneurysm, ApoE apolipoprotein E, MMP matrix metalloproteinase, Tregs regulatory T cells, BM-MSC bone marrow derived mesenchymal stem cells, IL-1 $\beta$ interleukin-1 $\beta$, EPA eicosapntemacnioc acid, DHA docosahexaenoic acid, TGF $\beta$ transforming growth facto, M-CSF macrophage colony-stimulating factor 


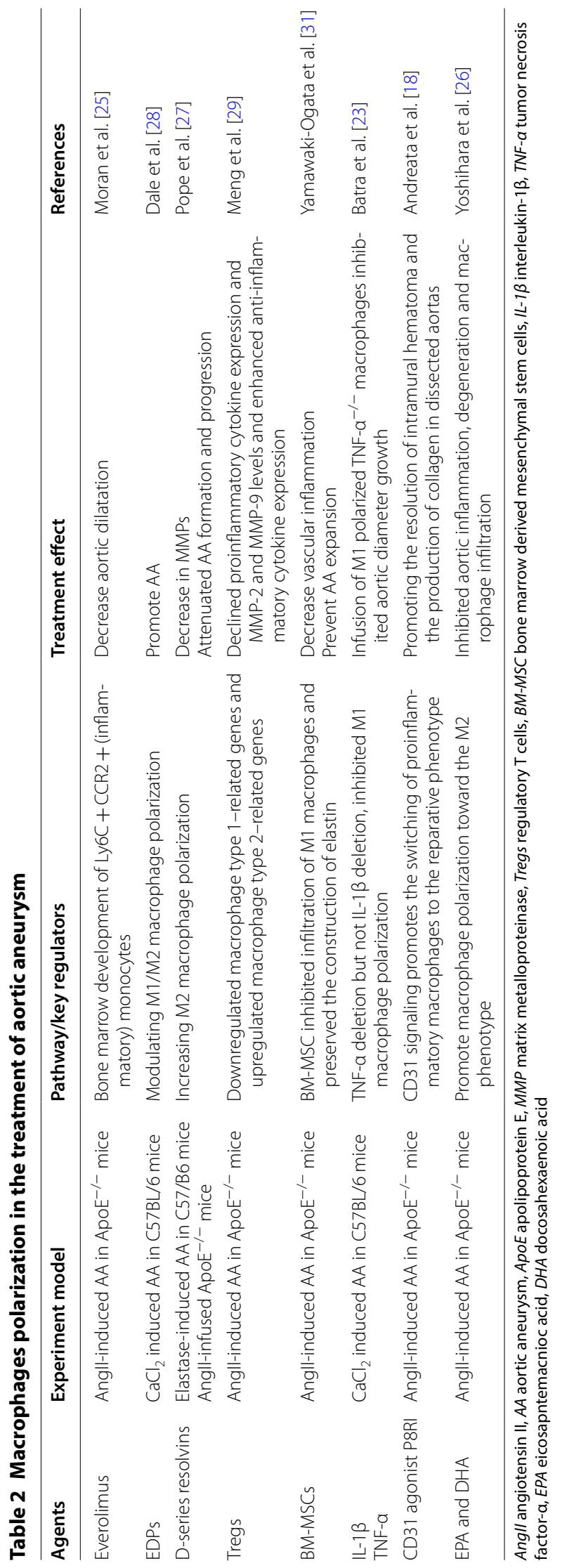


aneurysm induction. EDPs promoted a pro-inflammatory environment in aortic tissues by inducing M1 polarization, and neutralization of EDPs attenuated aortic dilation. Andreata et al. [18] found that CD31 agonist P8RI induces the switching of M1macrophages to the reparative M2 phenotype and promotes the healing of experimental dissected aortas in Apo $\mathrm{E}^{-/-}$mice with Ang II infusion. Meng et al. [29] reported that adoptive transfer of Tregs dose-dependently prevented AngII-induced AA in $\mathrm{ApoE}^{-1-}$ mice. One of the underlying mechanisms was that Tregs downregulated M1-related genes and upregulated M2-related genes, thus regulating macrophage polarization. Our group found that bone marrow derived mesenchymal stem cells (BM-MSCs) could regress the formation of AA by regulating macrophage polarization to restore the M1/M2 ratio and to reduce inflammation at the site of AA [30-32].

Taken together, these studies mentioned above demonstrate that the M1/M2 macrophage ratio plays a fundamental role in AA formation, development, and progression. Macrophage function can be stimulated or inhibited to improve healing and repair. The augmentation of aortic tissue repair by reducing M1 phenotype macrophage infiltration or promoting polarization to a reparative $\mathrm{M} 2$ phenotype seem be attractive therapeutic strategies for AA (Table 2).

\section{Summary and prospects}

In the present review, we summarized recently published studies on the roles of macrophage polarization in the aortic remodeling and development of AA. We highlighted the functions of macrophage subsets, which are complex, and could be either destructive or reparative during AA development. M1 macrophages accumulate in the aortic wall and dominate the cellular milieu and mainly clear cellular debris at early stage of AA. Thereafter, M1 macrophages secrete inflammatory cytokines that affect the consequent phases of aortic remodeling and initiate aortic tissue repair coordinated by M2 macrophages. The prolonged effects of M1 macrophages extend the destructive effects of inflammatory responses and cause expansion of AA. More recently, studies targeting macrophage differentiation towards the M2 phenotype have been shown to promote the resolution of aortic inflammation and slow AA progression. Modulation of macrophage subset polarization is believed to be an attractive strategy to prevent the progression of AA. However, macrophage polarization phenotypes are not always mutually exclusive, and it still remains unsolved whether some functional subsets represent real distinct populations. Additionally, the molecular and cellular mechanisms underlying regulation of macrophage polarization during AA development are complex and multifactorial. Further studies with more animal models and patients will be needed to be conducted to determine the precise roles of macrophage polarization in AA in order to develop effective treatment and prevention strategies.

\section{Authors' contributions}

$Z C$ and $X F$ have been involved in writing, compiling the manuscript. $Y Z, Y W$, QW and XL contributed significantly on literature and critical suggestions to reshape the manuscript. XF and XZ conceived the concept of this review, and revised it critically for publication standards. All authors read and approved the final version of manuscript.

\section{Author details \\ 1 Department of Hematology, Institute of Molecular Hematology, The Second Xiang-ya Hospital, Central South University, Changsha, Hunan, People's Republic of China. ${ }^{2}$ Department of Cardiovascular Surgery, The Second Xiang- ya Hospital, Central South University, Changsha, Hunan, People's Republic of China.}

\section{Acknowledgements}

The authors would like to thank Professor Hengyi Xiao (West China School of Mdicine/West China Hospital, Sichuan University, P.R.China) and Professor Guang-sen Zhang (Department of Hematology, Institute of Molecular Hematology, The Second Xiang-ya Hospital, Central South University, Changsha, Hunan, P.R. China) for their kindly support in this study.

Zhao Cheng-First Author.

\section{Competing interests}

The authors declare that they have no competing interests.

Availability of data and materials

All data generated or analysed during this study are included in this published article.

\section{Consent for publication}

Not applicable.

\section{Ethics approval and consent to participate}

This study was carried out in strict accordance with recommendations of the Regulations on human and animal experimentation of Central South University (China).

\section{Funding}

This work was financially supported by the National Natural Science Foundation of China (Grant No. 81400343), National Natural Science Foundation of China (Grant No. 81400093), \& Natural Science Foundation of Hunan Province, China (Grant No. 2018JJ3757).

\section{Publisher's Note}

Springer Nature remains neutral with regard to jurisdictional claims in published maps and institutional affiliations.

Received: 25 June 2018 Accepted: 6 December 2018 Published online: 13 December 2018

References

1. Lavingia KS, Dua A, Stern JR. Upper extremity access options for complex endovascular aortic interventions. J Cardiovasc Surg (Torino). 2018:59:360-7.

2. Chisci E, Alamanni N, lacoponi F, Michelagnoli S, Procacci T, Colombo G, Setacci C. Grading abdominal aortic aneurysm rupture risk. J Cardiovasc Surg (Torino). 2018;59:87-94.

3. Mascoli C, Vezzosi M, Koutsoumpelis A, lafrancesco M, Ranasinghe A, Clift P, Mascaro J, Claridge M, Adam DJ. Endovascular repair of acute 
thoraco-abdominal aortic aneurysms. Eur J Vasc Endovasc Surg. 2018;55:92-100

4. Sawyer DM, Pace LA, Pascale CL, Kutchin AC, O'Neill BE, Starke RM Dumont AS. Lymphocytes influence intracranial aneurysm formation and rupture: role of extracellular matrix remodeling and phenotypic modulation of vascular smooth muscle cells. J Neuroinflammation. 2016;13:185.

5. Forester ND, Cruickshank SM, Scott DJ, Carding SR. Functional characterization of T cells in abdominal aortic aneurysms. Immunology. 2005;115:262-70.

6. Forester ND, Cruickshank SM, Scott DJ, Carding SR. Increased natural killer cell activity in patients with an abdominal aortic aneurysm. Br I Surg. 2006:93:46-54

7. Hinterseher I, Schworer CM, Lillvis JH, StahI E, Erdman R, Gatalica Z, Tromp G, Kuivaniemi H. Immunohistochemical analysis of the natural killer cell cytotoxicity pathway in human abdominal aortic aneurysms. Int J Mol Sci. 2015:16:11196-212.

8. Sillesen H, Eldrup N, Hultgren R, Lindeman J, Bredahl K, Thompson M, Wanhainen A, Wingren U, Swedenborg J, Investigators AT. Randomized clinical trial of mast cell inhibition in patients with a medium-sized abdominal aortic aneurysm. Br J Surg. 2015;102:894-901.

9. Zhang L, Wang Y. B lymphocytes in abdominal aortic aneurysms. Atherosclerosis. 2015;242:311-7.

10. Murray PJ, Allen JE, Biswas SK, Fisher EA, Gilroy DW, Goerdt S, Gordon S, Hamilton JA, Ivashkiv LB, Lawrence T, et al. Macrophage activation and polarization: nomenclature and experimental guidelines. Immunity. 2014:41:14-20.

11. Tabas I, Bornfeldt KE. Macrophage phenotype and function in different stages of atherosclerosis. Circ Res. 2016;1 18:653-67.

12. Bories GFP, Leitinger N. Macrophage metabolism in atherosclerosis. FEBS Lett. 2017;591:3042-60.

13. Hamers AA, Vos M, Rassam F, Marinkovic G, Kurakula K, van Gorp PJ, de Winther MP, Gijbels MJ, de Waard V, de Vries CJ. Bone marrow-specific deficiency of nuclear receptor Nur77 enhances atherosclerosis. Circ Res. 2012;110:428-38.

14. Grinberg D, Pozzi M, Farhat F, Obadia JF. Ascending aortic aneurysm: update to existing guidelines. Presse Med. 2016;45:911-8.

15. Kulig P, Lewandowski K, Ziaja D, Zaniewski M, Kulig J. Endovascular aneurysm repair or open aneurysm repair for the treatment of abdominal aortic aneurysm — the latest update. Pol Przegl Chir. 2016;88:166-74.

16. Akerman AW, Stroud RE, Barrs RW, Grespin RT, McDonald LT, LaRue RAC, Mukherjee R, Ikonomidis JS, Jones JA, Ruddy JM. Elevated wall tension initiates interleukin-6 expression and abdominal aortic dilation. Ann Vasc Surg. 2018;46:193-204.

17. Zhang J, Chen H, Liu L, Sun J, Shi MA, Sukhova GK, Shi GP. Chemokine (C-C motif) receptor 2 mediates mast cell migration to abdominal aortic aneurysm lesions in mice. Cardiovasc Res. 2012;96:543-51.

18. Andreata F, Syvannarath V, Clement M, Delbosc S, Guedj K, Fornasa G, Khallou-Laschet J, Morvan M, Even G, Procopio E, et al. Macrophage CD31 signaling in dissecting aortic aneurysm. J Am Coll Cardiol. 2018;72:45-57.

19. Moore JP, Vinh A, Tuck KL, Sakkal S, Krishnan SM, Chan CT, Lieu M, Samuel CS, Diep H, Kemp-Harper BK, et al. M2 macrophage accumulation in the aortic wall during angiotensin II infusion in mice is associated with fibrosis, elastin loss, and elevated blood pressure. Am J Physiol Heart Circ Physiol. 2015;309:H906-17.
20. Boytard L, Spear R, Chinetti-Gbaguidi G, Acosta-Martin AE, Vanhoutte J, Lamblin N, Staels B, Amouyel P, Haulon S, Pinet F. Role of proinflammatory CD68(+) mannose receptor(-) macrophages in peroxiredoxin-1 expression and in abdominal aortic aneurysms in humans. Arterioscler Thromb Vasc Biol. 2013;33:431-8.

21. Hans CP, Koenig SN, Huang N, Cheng J, Beceiro S, Guggilam A, Kuivaniemi H, Partida-Sanchez S, Garg V. Inhibition of Notch1 signaling reduces abdominal aortic aneurysm in mice by attenuating macrophage-mediated inflammation. Arterioscler Thromb Vasc Biol. 2012:32:3012-23.

22. Hasan D, Chalouhi N, Jabbour P, Hashimoto T. Macrophage imbalance (M1 vs. M2) and upregulation of mast cells in wall of ruptured human cerebral aneurysms: preliminary results. J Neuroinflammation. 2012;9:222.

23. Batra R, Suh MK, Carson JS, Dale MA, Meisinger TM, Fitzgerald M, Opperman PJ, Luo J, Pipinos II, Xiong W, Baxter BT. IL-1 beta (Interleukin-1 beta) and TNF-alpha (tumor necrosis factor-alpha) impact abdominal aortic aneurysm formation by differential effects on macrophage polarization. Arterioscler Thromb Vasc Biol. 2018;38:457-63.

24. Wang SK, Xie J, Green LA, McCready RA, Motaganahalli RL, Fajardo A, Babbey CC, Murphy MP.TSG-6 is highly expressed in human abdominal aortic aneurysms. J Surg Res. 2017;220:311-9.

25. Moran CS, Jose RJ, Moxon JV, Roomberg A, Norman PE, Rush C, Korner $\mathrm{H}$, Golledge J. Everolimus limits aortic aneurysm in the apolipoprotein E-deficient mouse by downregulating C-C chemokine receptor 2 positive monocytes. Arterioscler Thromb Vasc Biol. 2013;33:814-21.

26. Yoshihara T, Shimada K, Fukao K, Sai E, Sato-Okabayashi Y, Matsumori R, Shiozawa T, Alshahi H, Miyazaki T, Tada N, Daida H. Omega 3 polyunsaturated fatty acids suppress the development of aortic aneurysms through the inhibition of macrophage-mediated inflammation. Circ J. 2015;79:1470-8.

27. Pope NH, Salmon M, Davis JP, Chatterjee A, Su G, Conte MS, Ailawadi G, Upchurch GR Jr. D-series resolvins inhibit murine abdominal aortic aneurysm formation and increase M2 macrophage polarization. FASEB J. 2016;30:4192-201.

28. Dale MA, Xiong W, Carson JS, Suh MK, Karpisek AD, Meisinger TM, Casale GP, Baxter BT. Elastin-derived peptides promote abdominal aortic aneurysm formation by modulating M1/M2 macrophage polarization. J Immunol. 2016;196:4536-43.

29. Meng X, Yang J, Zhang K, An G, Kong J, Jiang F, Zhang Y, Zhang C. Regulatory $T$ cells prevent angiotensin II-induced abdominal aortic aneurysm in apolipoprotein E knockout mice. Hypertension. 2014;64:875-82.

30. Fu XM, Yamawaki-Ogata A, Oshima H, Ueda Y, Usui A, Narita Y. Intravenous administration of mesenchymal stem cells prevents angiotensin IIinduced aortic aneurysm formation in apolipoprotein E-deficient mouse. J Transl Med. 2013;11:175.

31. Yamawaki-Ogata A, Fu X, Hashizume R, Fujimoto KL, Araki Y, Oshima H, Narita $Y$, Usui A. Therapeutic potential of bone marrow-derived mesenchymal stem cells in formed aortic aneurysms of a mouse model. Eur J Cardiothorac Surg. 2014;45:e156-65.

32. Yamawaki-Ogata A, Hashizume R, Fu XM, Usui A, Narita Y. Mesenchymal stem cells for treatment of aortic aneurysms. World J Stem Cells. 2014;6:278-87.

Ready to submit your research? Choose BMC and benefit from

- fast, convenient online submission

- thorough peer review by experienced researchers in your field

- rapid publication on acceptance

- support for research data, including large and complex data types

- gold Open Access which fosters wider collaboration and increased citations

- maximum visibility for your research: over $100 \mathrm{M}$ website views per year

At BMC, research is always in progress.

Learn more biomedcentral.com/submissions 